\title{
Organismos edáficos como bioindicadores da recuperação de solos degradados por arenização no Bioma Pampa
}

\author{
Edaphic organisms as bioindicators of restoration of degraded soils by arenização \\ on the Bioma Pampa
}

\author{
Ana Paula Moreira Rovedder ${ }^{\mathrm{I}}$ Flávio Luiz Foletto Eltz ${ }^{\mathrm{II}}$ Marta Sandra Drescher ${ }^{\mathrm{II}}$ \\ Ricardo Bergamo Schenato ${ }^{\text {III }}$ Zaida Inês Antoniolli'
}

RESUMO

\begin{abstract}
Os organismos edáficos, por sua sensibilidade a alterações no meio, têm sido utilizados como indicadores de modificações nos níveis de qualidade do solo, as quais podem ser promovidas por degradação ou agradação. Em Alegrete, Rio Grande do Sul, foram caracterizadas populações de organismos edáficos como bioindicadores dos efeitos da degradação por arenização e da recuperação por revegetação com Lupinus albescens Hook. \& Arn., fabácea natural do Bioma Pampa. Os tratamentos constituíram-se de solo com cobertura natural de Lupinus albescens (TN), área arenizada que recebeu revegetação com Lupinus albescens há um ano (T1), área arenizada que recebeu revegetação com Lupinus albescens há três anos (T3), solo arenizado (SA) e campo nativo (CN). As coletas foram feitas em fevereiro e maio de 2006, com armadilhas PROVID. Foram determinados os parâmetros: abundância de organismos em nível de Ordem, riqueza de organismos e índices de diversidade e igualdade de Shannon. A arenização reduziu o desenvolvimento das populações edáficas, enquanto a estratégia de revegetação mostrou efeitos positivos na recolonização da área. O grupo Collembola destacou-se como bioindicador dos efeitos dos processos de degradação e recuperação. O índice de diversidade de Shannon não foi adequado para a avaliação dos efeitos da arenização quando analisado isoladamente.
\end{abstract}

Palavras-chave: arenização, Lupinus albescens, bioindicadores, recuperação de solos.

\section{ABSTRACT}

Edaphic organisms are sensible to environment alterations and have been used as indicators of soil quality changes. These modifications can be promoted by degradation or agradation events. The study area is located in Alegrete, Rio Grande do Sul. It was analyzed the role of epigeic soil fauna as bioindicator of soil degradation by arenização and its restoration by revegetation with Lupinus albescens Hook. \& Arn., a native fabaceous of Bioma Pampa. The treatments were constituted of soil under natural covering with Lupinus albescens, degraded area recovered with Lupinus albescens by one year (T1), degraded area recovered with Lupinus albescens by three years (T3), degraded area (SA) and native grass field (CN). The sampling was made in February and May of 2006 with PROVID traps. It was determined the abundance of organisms at Order level, the organisms richness, the diversity and evenness Shannon' index. The process of Arenização reduced the development of sampled edaphic populations, while revegetation showed positive effects in the recolonization of the area. Colembolla group was a good bioindicator of degradation and restoration effects. The Shannon diversity index was not satisfactory to evaluate the edaphic fauna if disconnected of other parameters.

Key words: Lupinus albescens, bioindicators, soil restoration.

\section{INTRODUÇÃo}

Pode-se definir qualidade do solo como a capacidade deste em desempenhar funções dentro dos ecossistemas, como meio de suporte e desenvolvimento vegetal e de uma diversa biota faunística, atuando ainda como compartimento em

\footnotetext{
'Universidade Estadual do Rio Grande do Sul (UERGS), Pólo Cachoeira do Sul. Rua 7 de setembro, 1040, Centro, 96508-010, Cachoeira do Sul, RS, Brasil. E-mail: anarovedder@yahoo.com.br. Autor para correspodência.

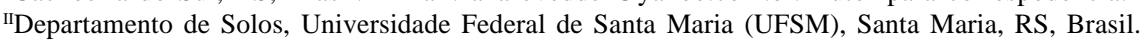

IIIPrograma de Pós-graduação em Ciência do Solo, UFSM, Santa Maria, RS, Brasil.
} 
ciclos biogeoquímicos importantes, tais como o ciclo da água e do carbono e como meio de descarte e retenção de elementos e resíduos (DORAN \& PARKIN, 1994). Ações de impacto negativo levam à degradação do ambiente edáfico e, conseqüentemente, ao comprometimento de suas funções dentro dos sistemas biológicos, enquanto a manutenção de índices elevados de qualidade do solo constitui-se em premissa para o estabelecimento da sustentabilidade em qualquer atividade humana.

Para se conhecer a magnitude dos efeitos impactantes, indicadores de qualidade do solo têm sido largamente utilizados em estudos comparativos (MERLIN, 2005; SANTOS et al., 2008), cujas informações produzidas podem colaborar para o desenvolvimento de estratégias de recuperação ou mitigação dos danos. Dentre tais indicadores, populações da pedofauna têm se destacado devido à sensibilidade a modificações no meio, respondendo a estas com relativa rapidez, comparativamente a outros indicadores de qualidade do solo como propriedades físicas e conteúdo de matéria orgânica do solo (REICHERT et al., 2003). Para ASSAD (1997), tal sensibilidade faz com que a compreensão do comportamento do solo em sistemas naturais ou antrópicos exija o conhecimento da pedofauna. Características como densidade e diversidade de populações de bioindicadores podem demonstrar as condições de um solo, seus níveis de equilíbrio ou perturbação (BROWN, 1997). Grupos funcionais da fauna edáfica podem desaparecer ou serem reduzidos em sua abundância e diversidade como resultado de processos de degradação do solo ou de substituição da diversidade natural por poucos organismos exóticos oportunistas e altamente adaptados a distúrbios (LAVELLE, 1996; LORANGER et al, 1999).

No sudoeste do Rio Grande do Sul, ocorrem solos de textura arenosa derivados de formações areníticas pós-vulcanismo da Formação Serra Geral, possivelmente do período Terciário (MEDEIROS et al., 2005), constituindo-se em um dos principais substratos do Bioma Pampa. Esses solos apresentam alta suscetibilidade aos processos erosivos, representados mais especificamente na região pelo fenômeno da arenização, termo proposto por SUERTEGARAY (1998) para o processo de retrabalhamento e exposição dos solos arenosos pelos agentes eólico e hídrico. Como conseqüência, ocorre perda da cobertura vegetal, por efeito abrasivo e soterramento, formando dunas ativas que se movimentam no sentido dos ventos predominantes (ROVEDDER et al., 2005). O inexpressivo grau de resiliência desse ecossistema leva ao aumento progressivo da degradação, uma vez instalado o núcleo de arenização. A rapidez com que ocorre esse processo requer estratégias eficientes para conter a expansão da arenização. Dentre estas, uma metodologia de revegetação com Lupinus albescens Horn. \& Arn, espécie de fabácea nativa do Bioma Pampa, tem demonstrado efeitos positivos como excelente produção de biomassa (ROVEDDER, 2007), redução da amplitude térmica do solo arenizado (ELTZ \& ROVEDDER, 2005) e recolonização da área afetada pela estrutura botânica do campo nativo (ROVEDDER, 2007).

Para avaliar o efeito da revegetação com Lupinus albescens no ambiente edáfico, foi desenvolvido um estudo comparativo tendo por base a abundância e diversidade de grupos epigéicos de organismos edáficos como bioindicadores da qualidade do solo. O presente trabalho apresenta uma análise da abundância de organismos, dos índices de diversidade e igualdade de Shannon, bem como da riqueza faunística, comparando-se diferentes coberturas do solo tomadas como tratamentos.

\section{MATERIAL E MÉTODOS}

A região de ocorrência da arenização no Rio Grande do Sul abrange o sudoeste do Estado, entre as latitudes de $29^{\circ} 00^{\prime} \mathrm{S}$ e $31^{\circ} 00^{\prime} \mathrm{S}$ e entre as longitudes de $54^{\circ} 30^{\prime} \mathrm{W}$ e $58^{\circ} 45^{\prime} \mathrm{W}$, com maior freqüência nos municípios de Alegrete, São Francisco de Assis, Itaqui, Cacequi, Quaraí e Manuel Viana(SUERTEGARAY, 1998). Para o presente estudo, escolheu-se um núcleo de arenização, campo nativo e área de ocorrência natural de Lupinus albescens. No núcleo de arenização, foram estabelecidas parcelas experimentais de revegetação com Lupinus albescens como estratégia de recuperação. Dessa forma, os tratamentos comparados foram "solo com cobertura natural de Lupinus albescens (TN)”, “área arenizada que recebeu revegetação com Lupinus albescens há três anos (T3)”, "área arenizada que recebeu revegetação com Lupinus albescens há um ano (T1)”, “campo nativo (CN)” e "solo degradado por arenização há 20 anos (SA)”. O tratamento TN constitui-se de área de ocorrência natural do Lupinus albescens, não sendo utilizada em sistemas produtivos. Os tratamentos T1 e T3 foram constituídos por parcelas experimentais instaladas sobre o solo arenizado há 20 anos, nas quais foi testada revegetação com Lupinus albescens. O plantio da espécie foi feito a lanço, sem revolvimento, com adubação proporcional a $120 \mathrm{~kg} \mathrm{ha}^{-1}$ de fósforo $\left(\mathrm{P}_{2} \mathrm{O}_{5}\right)$ e $120 \mathrm{~kg} \mathrm{ha}^{-1}$ de potássio $\left(\mathrm{K}_{2} \mathrm{O}\right)$ e $500 \mathrm{~kg} \mathrm{ha}^{-1}$ de calcário Filler, aplicados a lanço. $\mathrm{O}$ tratamento $\mathrm{CN}$ constitui-se de área com sistema de pecuária bovina. $\mathrm{O}$ tratamento SA era originalmente 
recoberto pelo campo nativo e manejado em sistema de pecuária bovina, sem nunca ter sido utilizado com cultivos agrícolas. Os tratamentos T1, T3, CN e SA são adjacentes, e TN distancia-se destes por $7 \mathrm{~km}$. Em todos os tratamentos o solo constitui-se de Neossolo Quartzarênico.

A tabela 1 apresenta a análise granulométrica, evidenciando-se o predomínio da fração areia em todos os tratamentos. A tabela 2 apresenta o conteúdo de carbono e macronutrientes encontrados em cada tratamento. A coleta dos organismos foi realizada em duas épocas de amostragem, sendo a primeira em fevereiro de 2006 e a segunda 60 dias depois, em maio de 2006. Como repetições, foram utilizadas sete armadilhas do tipo PROVID, conforme descrição de ANTONIOLLI et al. (2006) distribuídas aleatoriamente em cada tratamento. As aberturas das garrafas ficaram ao nível do solo para permitir a entrada dos organismos durante um tempo de permanência de quatro dias. Após esse período, as armadilhas foram retiradas. Os organismos foram separados dos resíduos por meio de peneiragem realizada com peneiras de abertura de 2 e $0,053 \mathrm{~mm}$. Os organismos foram classificados por ordem com auxílio de lupa biocular com aumento de 40 vezes.

Os parâmetros abundância, riqueza e índices de diversidade $(\mathrm{H})$ e igualdade $(\mathrm{E})$ de Shannon foram determinados por tratamento em cada época de coleta. A riqueza foi determinada de acordo com o número de ordens encontradas em cada tratamento. Os índices $\mathrm{H}$ e E de Shannon foram calculados conforme IBAÑEZ et al. (1995). A abundância total em cada tratamento dentro de cada época de coleta foi analisada estatisticamente pelo Teste t para populações independentes, em um nível de 5\% de probabilidade de erro.

\section{RESULTADOS E DISCUSSÃO}

A abundância nos tratamentos com vegetação (T3, T1, TN e CN) foi estatisticamente maior

Tabela 1 - Análise granulométrica (\%) para os tratamentos: revegetação com Lupinus albescens há três anos (T3), revegetação com Lupinus albescens há um ano (T1), área de ocorrência natural de Lupinus albescens (TN), campo nativo (CN) e solo arenizado (SA)

\begin{tabular}{|c|c|c|c|}
\hline & Argila & Silte & Areia \\
\hline & \multicolumn{3}{|c|}{ - } \\
\hline Т3 & 5,10 & 0,36 & 94,54 \\
\hline $\mathrm{T} 1$ & 6,30 & 0,80 & 92,90 \\
\hline $\mathrm{TN}$ & 5,24 & 0,84 & 93,92 \\
\hline $\mathrm{CN}$ & 6,3 & 0,80 & 92,90 \\
\hline SA & 4,30 & 0,36 & 95,34 \\
\hline
\end{tabular}

que no solo arenizado (SA) (Tabela 3), resultado relacionado à funcionalidade da cobertura vegetal em relação à quantidade e diversidade da biota edáfica (SILVAE CARVALHO, 2001; JANDL et al., 2003).

O tratamento TN apresentou a menor variação em abundância da primeira para a segunda coleta, demonstrando maior estabilidade no número de organismos da população, o que pode ser indício de um maior equilíbrio entre suas interações ecológicas (Tabela 3). O campo nativo (CN), embora também apresente a estrutura ecossistêmica natural, apresentou abundância significativamente menor que TN, T3 e T1, com grande variação entre as épocas de amostragem (Tabela 3). Provavelmente, a presença de pastejo nesse tratamento pode estar influindo na abundância de organismos, por atuar seletivamente sobre as espécies de campo e alterar o conteúdo de biomassa. Moço et al. (2005), comparando uma área de mata nativa com cultivo de eucalipto, capoeira e pastagem no norte fluminense, encontraram maior estabilidade na abundância de fauna edáfica ocorrendo na mata nativa. Avaliando a mesofauna em estudo comparativo entre cerrado nativo e três sistemas de uso do solo, Rodrigues et al. (1996) encontraram maior estabilidade na dinâmica de ocorrência de grupos na área de cerrado, com densidade e diversidade da fauna edáfica reduzidas nos sistemas de uso.

Já o solo arenizado (SA) apresentou a menor abundância nas duas épocas, com redução de 81,6\% da primeira para a segunda coleta, fato relacionado à perda de cobertura vegetal, o que tornou essa área menos propícia ao desenvolvimento das populações edáficas e à manutenção do seu equilíbrio populacional (Tabela 3).

Em relação à abundância por ordens, destacam-se as ordens Hymenoptera e Collembola, com elevada abundância nos tratamentos T3, T1, CN e TN, apresentando um comportamento importante como grupos representativos da fauna edáfica local. Estas apresentaram grande instabilidade populacional entre épocas de coleta. O número total de himenópteros, somando-se todos os tratamentos, diminuiu 59,47\%, e o número total de colêmbolos diminuiu $87,77 \%$ da primeira para a segunda coleta (Tabela 3).

Dentre os tratamentos, apenas o número de himenópteros em TN aumentou da primeira para segunda coleta. Nos demais casos, tanto himenópteros, quanto colêmbolos reduziram suas populações da primeira para segunda época de coleta. A temperatura do solo é uma propriedade afetada por perturbações no sistema (PREVEDELLO, 1996; HILLEL, 1998), como a perda de cobertura vegetal no processo de arenização. Sendo assim, a menor abundância em SA em relação 
Tabela 2 - Conteúdos de carbono (C), nitrogênio (N), potássio (K), cálcio (Ca), magnésio (Mg) e alumínio (Al) para os tratamentos: área com plantio de Lupinus albescens há três anos (T3), área com plantio de Lupinus albescens há um ano (T1), área de ocorrência natural de Lupinus albescens (TN), campo nativo (CN), solo arenizado (SA).

\begin{tabular}{|c|c|c|c|c|c|c|c|}
\hline & $\mathrm{C}$ & $\mathrm{N}$ & $\mathrm{P}$ & $\mathrm{K}$ & $\mathrm{Ca}$ & Mg & $\mathrm{Al}$ \\
\hline & \multicolumn{2}{|c|}{-----------g dm ${ }^{-3}----------$} & \multicolumn{2}{|c|}{----------mg dm'--------- } & \multicolumn{3}{|c|}{ - $\mathrm{cmol}_{\mathrm{c}} \mathrm{dm}^{-3}$} \\
\hline & & & & $-0-5 \mathrm{~cm}$ & & & ------ \\
\hline T3 & $0,75 d^{1}$ & $0,2 \mathrm{c}$ & $7,40 \mathrm{~b}$ & $11,39 \mathrm{~cd}$ & $0,10 \mathrm{c}$ & $0,12 \mathrm{~b}$ & 0,15 c \\
\hline $\mathrm{T} 1$ & $1,36 \mathrm{c}$ & $0,10 \mathrm{~d}$ & 13,56 a & 16,67 bc & $0,30 \mathrm{~b}$ & $0,12 \mathrm{~b}$ & $0,23 \mathrm{~b}$ \\
\hline TN & $3,04 \mathrm{~b}$ & $0,36 \mathrm{~b}$ & $8,31 \mathrm{~b}$ & 24,42 a & $0,70 \mathrm{a}$ & 0,35 a & $0,11 \mathrm{c}$ \\
\hline $\mathrm{CN}$ & 3,46 a & $0,33 \mathrm{~b}$ & $8,34 \mathrm{~b}$ & 21,38 a & $0,30 \mathrm{~b}$ & $0,05 \mathrm{c}$ & $0,16 \mathrm{c}$ \\
\hline SA & $0,57 \mathrm{~d}$ & $0,09 \mathrm{~d}$ & $4,16 \mathrm{c}$ & $8,52 \mathrm{~d}$ & $0,06 \mathrm{c}$ & $0,02 \mathrm{c}$ & 0,35 a \\
\hline T3 & $0,54 \mathrm{~d}$ & $0,10 \mathrm{~d}$ & $6,67 \mathrm{c}$ & $11,50 \mathrm{~b}$ & $0,10 \mathrm{c}$ & $0,06 \mathrm{~b}$ & $0,22 \mathrm{~b}$ \\
\hline $\mathrm{T} 1$ & $1,03 \mathrm{c}$ & $0,08 \mathrm{~d}$ & 14,22 a & 15,87 a & $0,30 \mathrm{a}$ & $0,01 \mathrm{c}$ & 0,32 a \\
\hline $\mathrm{TN}$ & $2,04 \mathrm{~b}$ & $0,21 \mathrm{c}$ & 7,91 b & $13,22 \mathrm{~b}$ & $0,20 \mathrm{~b}$ & 0,13 a & $0,28 \mathrm{ab}$ \\
\hline $\mathrm{CN}$ & $2,43 \mathrm{a}$ & $0,27 \mathrm{~b}$ & 7,72 b c & 15,78 a & 0,12 c & $0,04 \mathrm{~b}$ & $0,26 a b$ \\
\hline SA & $0,46 \mathrm{~d}$ & $0,08 \mathrm{~d}$ & $4,23 \mathrm{~d}$ & $5,40 \mathrm{c}$ & $0,10 \mathrm{c}$ & $0,01 \mathrm{c}$ & $0,24 a b$ \\
\hline
\end{tabular}

1 médias seguidas pela mesma letra na coluna, dentro de cada profundidade do solo, não diferem estatisticamente entre si pelo teste Duncan, em um nível de $5 \%$ de probabilidade de erro.

aos demais tratamentos também pode estar associada a maiores amplitudes térmicas devido à perda da cobertura vegetal. ELTZ \& ROVEDDER (2005), avaliando a temperatura do solo nas mesmas áreas de campo nativo e solo arenizado do presente trabalho, encontraram diferenças significativas, com o solo arenizado apresentando as maiores amplitudes térmicas.

Outras características ambientais agregamse e são significativas para a bioindicação da qualidade do solo. Para a ordem Collembola, por exemplo, sua posição na cadeia trófica, seu papel funcional nos

Tabela 3 - Abundância de organismos na primeira (fevereiro/2006) e na segunda coleta (maio/2006), por grupo de artrópodes edáficos e total em cada tratamento (T3=área com plantio de Lupinus albescens há três anos, T1=área com plantio de Lupinus albescens há um ano, TN=área de ocorrência natural de Lupinus albescens, C=campo nativo, SA=solo arenizado).

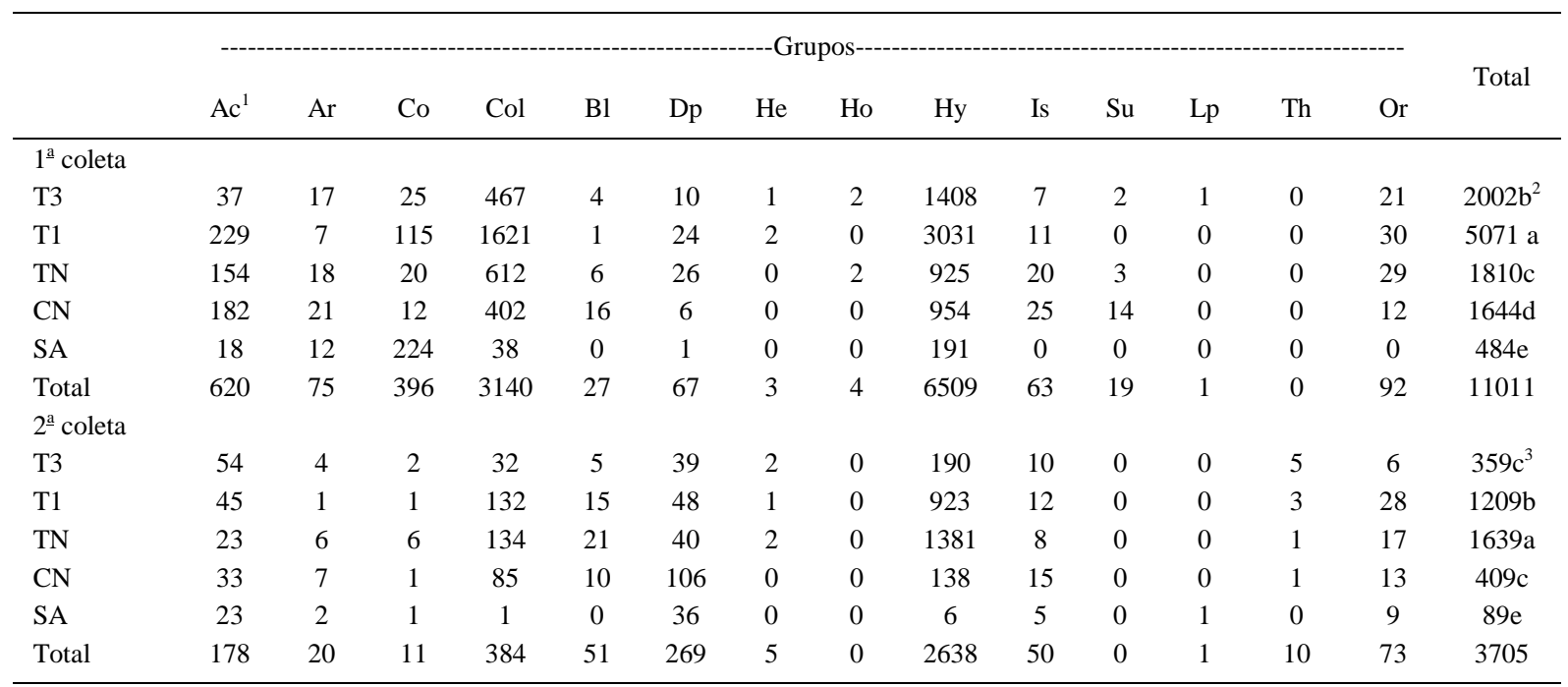

Ac ${ }^{1}$ : Acarina; Ar: Aranae; Co: Coleoptera; Col: Collembola; Bl: Blastodeae; Dp: Diptera; He: Hemiptera; Ho: Homoptera; Hy: Hymenoptera; Is: Isoptera; Su: Sulfugydae; Lp: Lepidoptera; Th: Thysanoptera; Or: Orthoptera. ${ }^{2}$. Valores seguidos pela mesma letra na coluna, para a primeira coleta, não diferem entre si pelo teste t de Student, em nível de $1 \%$ de probabilidade de erro ${ }^{3}$.Valores seguidos pela mesma letra na coluna, para a segunda coleta, não diferem entre si pelo teste t de Student, em nível de 1 \% de probabilidade de erro.

Ciência Rural, v.39, n.4, jul, 2009. 
processos que envolvem a dinâmica da matéria orgânica e sua sensibilidade de resposta a perturbações podem ser consideradas eficientes para um bom indicador de qualidade do solo (ASSAD, 1997; CROSSLEY \& COLEMAN, 2000; COLEMAN \& HENDRIX, 2000). Esses aspectos tornam-se relevantes quando se observa a diferença na abundância de Collembola entre os tratamentos com cobertura vegetal e o tratamento com solo arenizado (SA), com uma drástica redução do grupo neste último (Tabela 3). LORANGER et al. (1999) também encontrou redução da população e da riqueza de espécies de colêmbolos em sítios que perderam sua cobertura vegetal.

Já a ordem Hymenoptera constitui-se em um dos mais importantes grupos em solos tropicais e subtropicais devido à abundância, à diversidade e aos aspectos funcionais, com influência em aspectos pedológicos e na regulação do equilíbrio ecológico (ASSAD, 1997). Quando esses solos são perturbados, esse grupo é capaz de manifestar alterações em sua população que expressem tais perturbações. A predominância de Hymenoptera em todos os tratamentos (Tabela 3) indicou tendência a baixos índices de diversidade no ecossistema (Tabela 4), fato que pode estar relacionado à baixa fertilidade natural do solo em estudo. A população de Hymenoptera em TN aumentou da primeira para a segunda coleta, contrariamente à tendência geral do experimento, o que reduziu os índices de diversidade $(\mathrm{H})$ e de equitabilidade (J) de TN na segunda coleta. Nessa época de amostragem, Diptera destacou-se, aumentando sua abundância em todos os tratamentos. Enquanto a

Tabela 4 - Índice de diversidade de Shannon (H), índice de igualdade de Shannon (E), índice de Riqueza (S) em revegetação com Lupinus albescens há três anos (T3), revegetação com Lupinus albescens há um ano (T1), área de ocorrência natural de Lupinus albescens (TN), campo nativo (CN) e solo arenizado (SA).

\begin{tabular}{llll}
\hline & $\mathrm{S}$ & $\mathrm{H}$ & $\mathrm{E}$ \\
\hline $1^{\mathrm{a}}$ coleta & & & \\
$\mathrm{T} 3$ & 13 & 0,88 & 0,34 \\
$\mathrm{~T} 1$ & 10 & 0,98 & 0,43 \\
$\mathrm{TN}$ & 11 & 1,22 & 0,51 \\
$\mathrm{CN}$ & 10 & 1,20 & 0,52 \\
$\mathrm{SA}$ & 6 & 1,15 & 0,64 \\
$2^{\mathrm{a}}$ coleta & & & \\
T3 & 12 & 1,57 & 0,63 \\
T1 & 11 & 0,92 & 0,38 \\
TN & 11 & 0,68 & 0,28 \\
CN & 10 & 1,26 & 0,55 \\
SA & 10 & 1,73 & 0,75 \\
\hline
\end{tabular}

Ordem Sulfugyda desaparece na segunda coleta, Thysanopera aparece somente nessa coleta (Tabela 3).

Os baixos valores de diversidade e igualdade de Shannon para os tratamentos T3, T1 (primeira e segunda coleta) e TN (segunda coleta) (Tabela 4) representam a desproporcionalidade entre grupos edáficos, exemplificada pelo predomínio de Hymenoptera e Collembola, o que está relacionado com a maior abundância encontrada nesses tratamentos (Tabela 3). Uma vez que o conceito de diversidade refere-se à variedade de organismos vivos e combina riqueza e uniformidade (ASSAD, 1997), a menor igualdade entre grupos reduziu o índice de diversidade. Na segunda coleta, T3 elevou consideravelmente seus índices de diversidade e igualdade devido à drástica redução no número total de indivíduos (Tabela 3), fato que aumentou a equitabilidade entre grupos (Tabela 4). Aspectos como maior abundância e riqueza no solo arenizado que recebeu revegetação (tratamentos T3 e T1) podem ser decorrentes da amenização dos efeitos da arenização como abrasão e soterramento e diminuição da amplitude térmica devido à entrada de insumos no momento do plantio do Lupinus albescens, disponibilizando uma fonte de nutrientes mais rica do que a encontrada originalmente nos campos nativos da região. De acordo com RAPAPORT (1968), há uma relação direta entre fertilidade do solo e abundância da fauna edáfica. Como a revegetação em T1 é mais recente, o efeito residual da adubação foi mais intenso, principalmente em relação ao conteúdo de fósforo no solo (Tabela 1), o que se refletiu na abundância de organismos.

Relacionando abundância (Tabela 3) com diversidade e igualdade de Shannon (Tabela 4), todos os tratamentos, exceto solo arenizado (SA), mostraram grande desuniformidade entre grupos. SA apresentou maiores valores para igualdade nas duas épocas de coleta devido à proporcionalidade entre baixos valores de riqueza e baixos valores de abundância nesse tratamento. O índice H é máximo para alguma riqueza (S) se os objetos do estudo (no caso os tratamentos) têm igual número de indivíduos; e se aproxima de seu valor mínimo se os indivíduos apresentarem maior concentração em um objeto, ou seja, quando tiverem menor igualdade (IBÁÑEZ et al., 1995). Portanto, quanto maior a igualdade entre grupos, maior o índice de diversidade. Dessa forma, os tratamentos que apresentaram o predomínio de organismos em um grupo, notadamente Colembolla e Hymenoptera, reduziram sua igualdade entre grupos e, conseqüentemente, apresentaram menores índices de diversidade. Essa relação inversamente proporcional 
entre abundância e índice de diversidade é comum devido à dominância de grupos. ANTONY (2000) encontrou a mesma disparidade quando comparou a fauna edáfica em área de floresta e área de floresta perturbada por queima na Amazônia.

Em relação aos valores de riqueza, observase que SA apresentou o menor dos valores encontrados na primeira coleta (Tabela 4). A riqueza de fauna edáfica também está relacionada com a disponibilidade e qualidade de cobertura vegetal como fonte de nutrientes e abrigo. A reduzida abundância e a riqueza em SA configuraram-se em uma distribuição mais uniforme do número de indivíduos por ordem, implicando maiores índices $\mathrm{H}$ e E, principalmente na segunda coleta. Assim, poder-se-ia concluir que SA é mais biodiverso e que a arenização, portanto, afeta mais o parâmetro abundância do que riqueza e diversidade. Esse fato poderia levar a uma interpretação de que esse ambiente encontra-se em maior equilíbrio.

Contudo, a avaliação de sua abundância total demonstra que esta é muito baixa em relação às possibilidades apresentadas pelos tratamentos com vegetação, não permitindo um amplo desenvolvimento das populações da fauna edáfica e, dessa forma, não contemplando todos os nichos funcionais inerentes a esta. Sendo assim, muitos grupos funcionais desses organismos estão excluídos da área arenizada (SA). De acordo com TÓTOLA \& CHAER (2002), as análises de riqueza, diversidade e igualdade devem indicar se os aspectos funcionais da biodiversidade variam entre diferentes locais ou entre diferentes habitats em um mesmo local. Sendo assim, o aspecto funcionalidade das comunidades de artrópodes edáficos não está amplamente contemplado pelo índice $\mathrm{H}$ nesse caso, uma vez que a baixa riqueza e a abundância apresentadas pelo tratamento SA não proporciona a plena ocupação dos possíveis nichos ecológicos. Quando ocorre redução na integridade ecológica de um ecossistema, ou seja, na sua capacidade de manter um potencial evolutivo por longo prazo, a capacidade de resistir a impactos torna-se menor. Cada comunidade de um ecossistema responderá diferentemente a esses impactos, diferindo no grau de sensibilidade, de resposta e de recuperação (PINHEIRO, 2004).

Portanto, os índices H e E obtidos em SA podem estar demonstrando que, apesar da estrutura ecossistêmica em solos arenizados estar degradada (houve a perda total da vegetação de campo nativo), esse fato não foi suficiente para causar pressão de seleção em determinados grupos. Esse fato vai ao encontro da teoria de que a arenização é um fator natural (SUERTEGARAY, 1998), podendo apresentar adaptação evolutiva de organismos que povoaram a região. Com isso poder-se-ia pressupor que não devem ser realizadas intervenções na tentativa de recuperar a área de campo perdida com a arenização, mantendo-se os areais intactos. Contudo, deve-se ressaltar que, uma vez instalado o areal, este tende a se expandir indefinidamente, pois o ecossistema apresenta baixa resiliência em relação ao efeito da arenização, não conseguindo resistir ao soterramento e ao efeito abrasivo das partículas em suspensão, ambos efeitos da movimentação de areia.

A manutenção das áreas arenizadas, portanto, implica também em perdas da diversidade local dos campos regionais, além da perda da capacidade produtiva destes. Implica ainda em modificações no ambiente edáfico, já naturalmente frágil, como alterações no regime hídrico e na amplitude térmica. Nessa situação, a revegetação com espécies próprias do ecossistema local (como o Lupinus albescens) traria benefícios como conservação da diversidade genética local, da qualidade do solo e de sua produtividade (ROVEDDER, 2007), sem interferir na dinâmica natural do ecossistema. Depreende-se dessa avaliação que o índice de diversidade $\mathrm{H}$ não foi suficiente para a determinação da qualidade de um solo arenizado, quando analisado isoladamente. A diversidade de invertebrados edáficos resulta na produção de estruturas, cuja abundância e qualidade são fundamentais para a conservação e dinâmica da matéria orgânica do solo, ciclagem de nutrientes e manutenção de propriedades físicas, essenciais para a sustentabilidade da produção primária (LAVELLE, 1996). Portanto, reduções na sua abundância tal como ocorre no solo arenizado são prejudiciais à manutenção do ecossistema, mesmo com um nível elevado de adaptação por parte dos organismos.

\section{CONCLUSÃO}

A fauna edáfica foi um indicador eficiente da influência da presença ou ausência de cobertura vegetal, tendo sido influenciada pela época de coleta, presença ou ausência de cobertura vegetal e tipo de cobertura vegetal. A arenização reduziu o desenvolvimento das populações edáficas amostradas, contribuindo para a degradação do compartimento biológico do solo. Os grupos Collembola e Hymenoptera foram bons bioindicadores dos efeitos da arenização e da variação entre tratamentos.

Para solos sujeitos à arenização, o índice de diversidade de Shannon não foi adequado para a observação dos aspectos funcionais desempenhados pelas populações de artrópodes edáficos. A complexidade do processo de arenização e a incipiência 
dos estudos em torno do tema indicam a importância de uma maior atenção por parte da pesquisa científica, necessitando de abordagens investigativas mais freqüentes e aprofundadas para melhor entendimento da complexidade desse processo e elaboração de planos de recuperação.

\section{REFERÊNCIAS}

ANTONIOLLI, Z.I. et al. Método alternativo para estudar a fauna do solo. Ciência Florestal, Santa Maria, v.16, n.4, p.407-417, 2006

ANTONY, L.M.K. Abundância e distribuição vertical da fauna do solo de ecossistemas amazônicos naturais e modificados. In: REUNIÃO BRASILEIRA DE FERTILIDADE DO SOLO E NUTRIÇÃO DE PLANTAS, 25., REUNIÃO BRASILEIRA SOBRE MICORRIZAS 8., SIMPÓSIO BRASILEIRO DE MICROBIOLOGIA DO SOLO 6., REUNIÃO BRASILEIRA DE BIOLOGIA DO SOLO, 3., 2000, Santa Maria, RS. Anais... Santa Maria: SBCS/SBM/UFSM, 2000. 1 CD-ROM.

ASSAD, M.L.L. Fauna do solo. In: VARGAS, M.A.T. \& HUNGRIA, M. (Eds.). Biologia dos solos dos Cerrados. Planaltina: Embrapa, 1997. p.363-443.

BROWN, K.S. Insetos como rápidos e sensíveis indicadores de uso sustentável dos recursos naturais. In: MARTOS, H.L.; MAIA, N.B. (Ed.). Indicadores ambientais. Sorocaba: PUC / Shell Brasil, 1997. p.143-151.

COLEMAN, D.C.; HENDRIX, P.F. Invertebrates as webmasters in ecosystems. London: CABI Publishing, 2000. 336p.

CROSSLEY, D.A.; COLEMAN, D.C. Microarthropods. In: SUMMER, M.E. Handbook of soil science. New York: CRC, 2000. p.C59-C85.

DORAN, J.W.; PARKIN, T.B. Defining and assessing soil quality. In: DORAN, J.W. et al. (Eds). Defining soil quality for a sustenaible environment. Madison: Soil Science Society of America, 1994. p.3-21. (Special publication, 35).

ELTZ, F.L.F.; ROVEDDER, A.P.M. Revegetação e temperatura do solo em áreas degradadas no sudoeste do Rio Grande do Sul. Revista Brasileira de Agrociência, v.11, n.2, p.193-200, 2005.

HILLEL, D. Environmental soil physics. Nova York: Academic, 1998. 771p.

IBÁÑEZ, J.J. et al. Pedodiversity: concepts and measures. Catena, n.24, p.215-232, 1995. Disponível em: http:// www.sciencedirect.com/science?_ob=ArticleURL\&_udi=B6VCG3YCN43Y-9\&_user $=687358 \& \_r d o c=18 \_f m t=8 \_$orig $=$search $\&$ \& sort $=$ d\& $\&$ view $=c \&$ _acct $=C 000037899 \&$ _versio

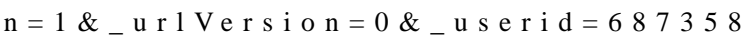
\&md5=f3aeb610ad7787391bf5fa58a1cb3bd2. Doi: 10.1016/ 0341-8162(95)00028-Q.
JANDL, R. et al. Forest soil chemistry and mesofauna 20 years after an amelioration fertilization. Restoration Ecology, v.11, n.2, p.239-246, 2003. Disponível em: http:// www3.interscience.wiley.com/journal/118893189/ abstract?CRETRY $=1 \&$ SRETRY $=0$. Doi: 10.1046/j.1526100X.2003.00179.x.

LAVELLE, P. Diversity of soil fauna and ecosystem function. Biology International, n.33, p.3-15, 1996

LORANGER, G. et al. Influence of agricultural practices on arthropod communities in a vertisol (Martinique). European Journal of Soil Biology, v.34, n.3, p.157-165, 1999. Disponível em: http:// www.sciencedirect.com/science?_ob=ArticleURL\&_udi=B6VR7$3 Y$ YXN51-2\&_user $=687358 \&$ \&doc $=1 \&$ \& $\mathrm{mt}=$ \&_orig $=$ se a r c h \& _sort $=$ d \& vi e w $=$ c \& _ a c c t $=$ C 00003 $7899 \&$ \& version $=1 \&$ \& url Version $=0 \&$ \& use rid=687358\&md5=369528dd359fd58f497f981f721069af. Doi: 10.1016/S1164-5563(00)86658-3.

MEDEIROS, E. et al. Degradação ambiental na região centrooeste do Rio Grande do Sul. Ciência \& Ambiente, v. 11, p.53-64, 2005.

MERLIM, A.O. Macrofauna edáfica em ecossistemas preservados e degradados de araucária no Parque Estadual de Campos do Jordão, SP. 2005. 89f. Dissertação (Mestrado em Ecologia de agroecossistemas) - Escola Superior de Agricultura Luiz de Queiroz.

MOÇO, M.K.S. et al. Caracterização da fauna edáfica em diferentes coberturas vegetais na região norte fluminense._ Revista Brasileira de Ciência do Solo, v. 29, n. 6, p.555-564, 2005. Disponível em: http://www.scielo.br/scielo.php?script=sci_arttext\&pid=S010006832005000400008\&lng=en\&nrm=iso\&tlng=pt. Doi: 10.1590/ S0100-06832005000400008.

PINHEIRO, A. Monitoramento e avaliação da qualidade das águas. In: ROMEIRO, A.R. (Org.). Avaliação e contabilização de impactos ambientais. Campinas: UNICAMP, 2004. p. 172-182.

PREVEDELlo, C.L. Física do solo: com problemas resolvidos. Curitiba: Salesward-Discovery 1996. 446p.

RAPAPORT, E.H. La fauna edáfica y sus aplicaciones en la caracterización de los suelos. In: CONGRESSO LATINOAMERICANO DE BIOLOGIA DO SOLO, 2., 1968, Santa Maria. Anais... Santa Maria: Universidade Federal de Santa Maria, Instituto de Solos e Culturas, 1968. p.155-173.

REICHERT, J.M. et al. Qualidade do solo e sustentabilidade de sistemas agrícolas. Ciência \& Ambiente, v.27, p.29-48, 2003.

RODRIGUES, L.O. et al. Avaliação da fauna do solo sob vários sistemas de manejo em um latossolo da região dos Cerrados. In: SIMPÓSIO SOBRE CERRADO, 8. INTERNATIONAL SYMPOSIUM ON TROPICAL SAVANNAS, 1996, Planaltina. Anais... Planaltina: Embrapa-CPAC, 1996. p.375-378.

ROVEDDER, A.P.M. et al. Análise da composição florística do campo nativo afetado pelo fenômeno da arenização no sudoeste 
do Rio Grande do Sul. Revista Brasileira de Agrociência, v.11, n.4, p.501-503, 2005.

ROVEDDER, A.P.M. Potencial do Lupinus albescens Hook. \& Arn. para recuperação de solos arenizados do Bioma Pampa. 2007. 126f. Tese (doutorado em Ciência do Solo) Programa de Pós-graduação em Ciência do Solo, Universidade Federal de Santa Maria.

SANTOS, GG. et al. Macrofauna edáfica associada a plantas de cobertura em plantio direto em um Latossolo Vermelho do Cerrado. Pesquisa Agropecuária Brasileira, v.43, n.1, p.115-122, 2008. Disponível em: http://www.scielo.br/scielo.php?script=sci_arttext\&pid=S0100204X2008000100015\&lng=pt\&nrm=iso\&tlng=pt. Doi: 10.1590/ S0100-204X2008000100015.
SILVA, R.A.; CARVALHO, G.S. Ocorrência de insetos na cultura do milho em sistema de plantio direto, coletados com armadilhas de solo. Ciência Rural, v.30, n.2, p.199-203, 2001. Disponível em: http://www.scielo.br/scielo.php?script=sci_arttext\&pid=S0103$84782000000200001 \& \operatorname{lng}=$ en\&nrm=iso\&tlng=pt. Doi: 10.1590/ S0103-84782000000200001.

SUERTEGARAY, D.M.A. Deserto grande do sul: controvérsia. 2.ed. Porto Alegre: UFRGS, 1998. 130p.

TÓTOLA, M.R.; CHAER, G.M. Microorganismos e processos microbiológicos omo indicadores da qualidade dos solos. In: SOCIEDADE BRASILEIRA DE CIÊNCIA DO SOLO. (Ed.). Tópicos em Ciência do Solo, Viçosa: Sociedade Brasileira de Ciência do Solo, 2002. V.2, p.195-276. 